\title{
Successful use of Ustekinumab in a Patient with Psoriasis, Psoriatic Arthritis and Systemic Lupus Erythematosus: A Case report and Review of literature
}

\author{
Aman Kugasia ${ }^{1}$, Chandrahasa Annem ${ }^{2}$, Palashkumar Jaiswal ${ }^{3}$, Ailda Nika $^{1}$, Winston Sequeira ${ }^{1}$, Joel \\ Block $^{1}$ and Meenakshi Jolly ${ }^{1 *}$ \\ ${ }^{1}$ Department of Rheumatology, Rush University Medical Center, USA
}

${ }^{2}$ Presbyterian Medical Center, Albuquerque, USA

${ }^{3}$ Department of Internal Medicine, John H Stroger Jr. Hospital of Cook County, USA

Received: December 07, 2017; Published: December 15, 2017

*Corresponding author: Meenakshi Jolly, Rush University Medical Center, University Rheumatologist 1611 West Harrison Street, Chicago- IL- 60612, Tel: 312-563-2800; Email: meenakshi_jolly@rush.edu

\begin{abstract}
Studies have demonstrated the role of TH-17 pathway in the pathogenesis of Systemic Lupus Erythematosus (SLE). Ustekinumab, a monoclonal antibody, binds and inhibits p 40 subunit of IL-12 and 23, leading to IL-17 blockage, causing inhibition of TH-17 signaling pathway. Ustekinumab has rarely been used in the treatment of SLE. Herein, we discuss a patient with long standing history of Psoriasis and Psoriatic Arthritis, which was successfully being managed with anti TNF therapy. He was later diagnosed to have concomitant SLE. His persistently active and aggressive psoriatic lesions, arthritis and thrombocytopenia posed an interesting therapeutic dilemma. Fortunately his psoriatic lesions, arthritis and thrombocytopenia responded well to ustekinumab. Methotrexate was added to his regimen after improvement and he remained in remission on this therapy. Further clinical studies are ongoing to investigate the role of TH-17 blocking agents in SLE.
\end{abstract}

Keywords: Ustekinumab; Systemic Lupus Erythematosus (SLE); Psoriasis; Thrombocytopenia; IL 17; IL 23

Abbreviations: SLE: Systemic Lupus Erythematosus; CCLE: Chronic Cutaneous Lupus Erythematosus; DLE: Discoid Lupus Erythematosus; PsA: Psoriatic Arthritis; Ps: Psoriasis; TNF: Tumor Necrosis Factor; IL: Interleukin; AIHA: Auto Immune Hemolytic Anemia; PUV-A: Photo Ultraviolet A; Anti Ds-DNA: Anti-Double Stranded Deoxyribonucleic Acid; LAC: Lupus Anticoagulant; Anti RNP: Anti- Ribo Nuclear Protein; Anti Sm: Anti- Smith; MRI: Magnetic Resonance Imaging; Mg: Milligrams

\section{Introduction}

Interleukins (IL) are involved in the pathogenesis of several autoimmune disorders such as rheumatoid arthritis, crohn's disease and psoriasis (Ps), and specific IL blockade has been utilized successfully to treat patients with these diseases [1-2]. The role of the p40 subunits of IL-12 and IL-23 is increasingly being explored in the pathogenesis of systemic lupus erythematous (SLE) [3-7]. Ustekinumab is a human monoclonal antibody that binds and inhibits the p40 subunit of interleukins 12 and 23, which are involved in the TH-17 signaling pathway. It is approved for the treatment of moderate to severe plaque Psoriasis and psoriatic arthritis[8]. While there have been isolated case reports of its successful use for the treatment of refractory cutaneous lupus (non SLE), suggesting that targeting the TH-17 pathway may be useful in this condition, its use in SLE remains poorly described [9-10]. Here, we report a case of successful use of Ustekinumab in a patient with active SLE and psoriasis (Ps).

\section{Case Report}

Our patient is a 48-year-old man with history of psoriasis (Diagnosed in 2001), autoimmune hemolytic anemia (AIHA) treated with rituximab (diagnosed in 2006 and rituximab given in 2006), who presented to the rheumatology clinic (2008) for management of psoriatic arthritis (PsA). His psoriasis had only partially responded to topical isotretinoin and PUV-A. Physical examination showed erythematous, scaly plaques involving the lower extremities, lower back, and the extensor surfaces of both elbows. His left knee was warm, tender, and non-erythematous with a moderate sized effusion. Synovial fluid analysis showed $18,000 \mathrm{wbc} / \mu \mathrm{L}$ (87\% neutrophils) without crystals. Gram stain of synovial fluid stain was negative. Laboratory evaluation showed thrombocytopenia $(52,000$ cells $/ \mu \mathrm{L})$, positive anti-nuclear antibody (ANA) (1:640), anti-double stranded DNA antibody (Anti Ds-DNA) (42 IU), anti SS-B antibodies at 1.18 ( $\geq 1.10$ is positive) 
and lupus anticoagulant (LAC). Anti ribonuclear protein (RNP), anti-Smith (Sm), anti-histones, anti-SS-A, anti $\beta$ - 2 glycoprotein and anti cardiolipin antibodies were negative. His white cell count was at 9200 cells $/ \mu \mathrm{L} \&$ hemoglobin was stable at $14 \mathrm{~g} / \mathrm{dL}$.

Comprehensive metabolic panel, complements and urinalysis were within normal range. Knee radiograph revealed mild narrowing of the lateral patello-femoral compartment. MRI of the right knee showed mild enthesopathy of anterior patella). A diagnosis of psoriasis, psoriatic arthritis (oligo-articular asymmetric arthritis pattern with inflammatory synovial fluid) was made. Patient was started on etanercept 50mg every week subcutaneously (5/2010). His skin disease and arthritis did not improve after 12 months on this medication so he was switched to infliximab 500mg every two weeks subcutaneously. In retrospect he was diagnosed with SLE clinically based on positive ANA, anti-Ds-DNA, positive lupus anticoagulant, AIHA that responded to rituximab and thrombocytopenia. Concern for the druginduced lupus was low because AIHA preceded any biological agent administration and thrombocytopenia preceded infliximab administration. At this point fliximab was discontinued on the side of caution. He was substituted to mycophenolate mofetil. Patient's skin lesions, synovitis of knees and platelets did not improve after 6 months therapy with mycophenolate 1500 twice a day and prednisone $10 \mathrm{mg}$ daily. He was switched to cyclosporine $300 \mathrm{mg}$ daily along with prednisone.

This regimen improved his psoriatic skin lesion (Figure 1) but he had persistent arthritis, recurrent inflammatory knee effusions and low platelets. We initiated ustekinumab $90 \mathrm{mg}$ subcutaneously at 0,4 week and then every 12 weeks and cyclosporine was stopped. After three doses of ustekinumab, there was complete resolution of skin lesions and normalization of platelet count $(200,000$ cells $/ \mu \mathrm{L}$ ). Methotrexate $10 \mathrm{mg}$ every week was added given persistent arthritis. After four months of therapy with ustekinumab and methotrexate, prednisone was tapered off. Patient had been on the current regimen for the last five years without recurrence of thrombocytopenia, AIHA, psoriatic skin lesion or arthritis. No medication related adverse effects have been noted. Timeline of events have been depicted in chart form in Figure 2.

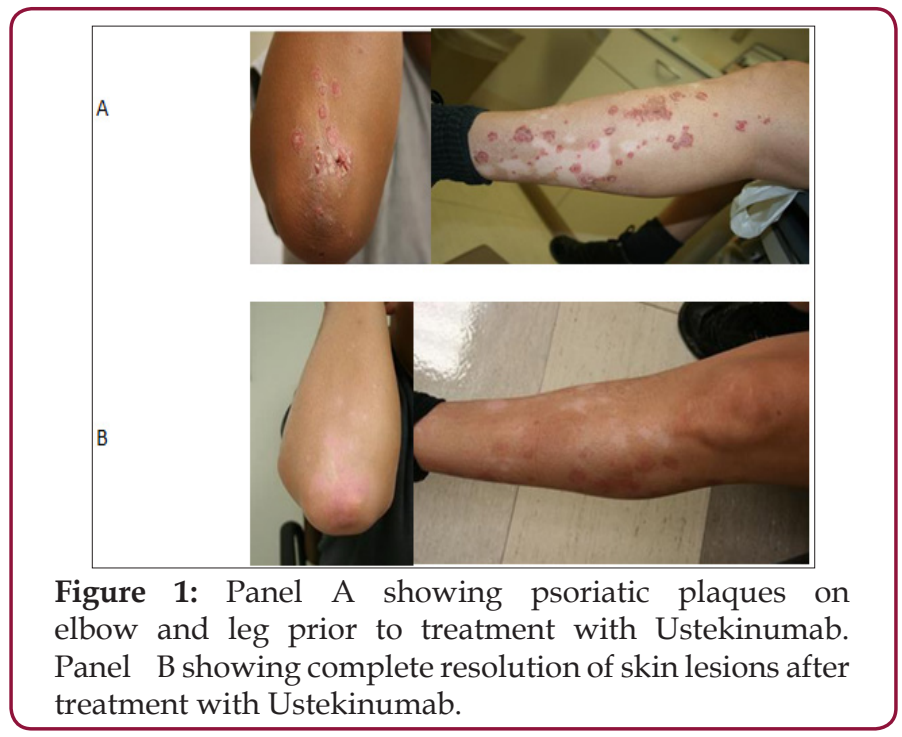

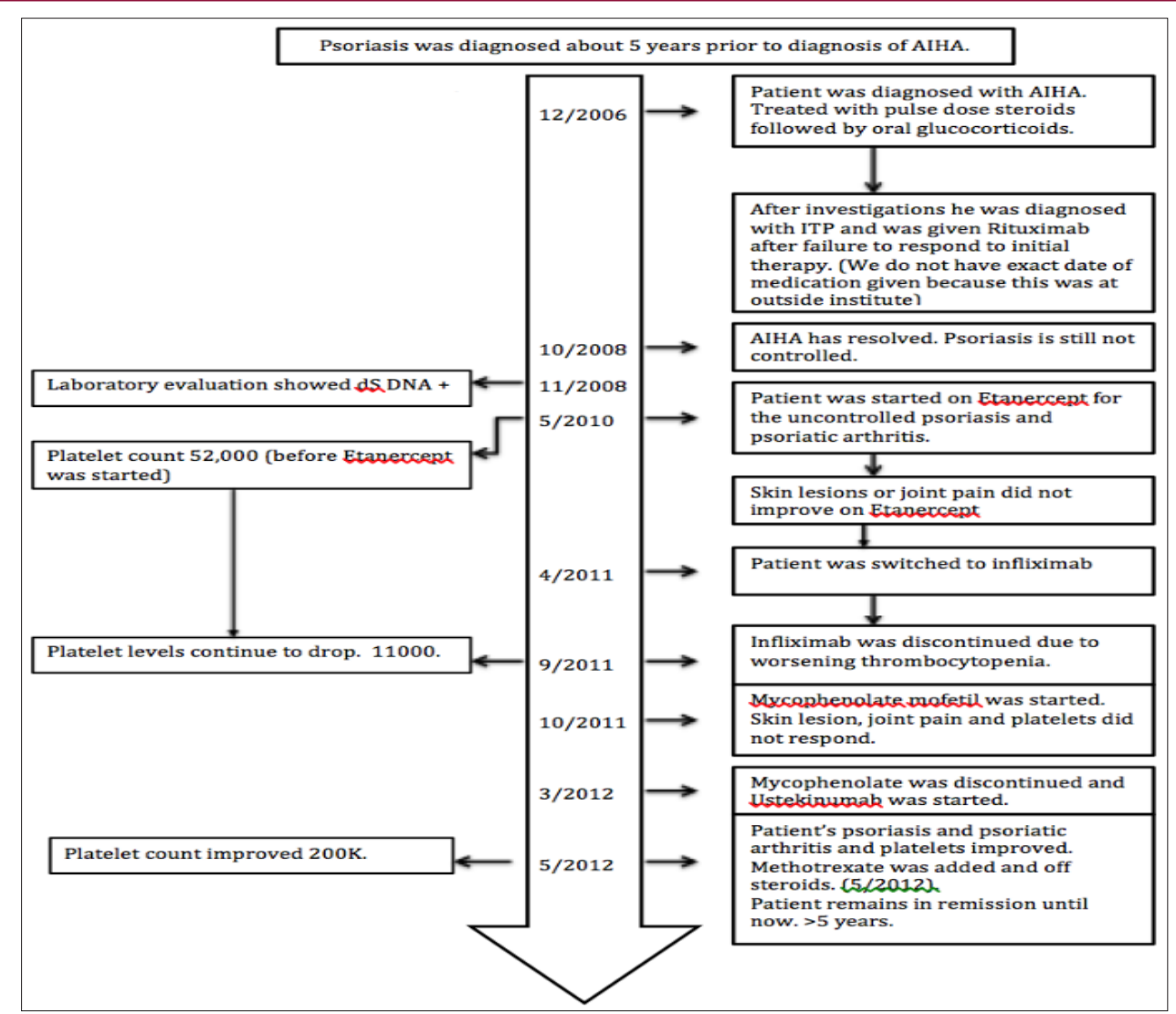

Figure 2: Timeline for the events. 


\section{Methods and Materials}

This systematic review was conducted according to the PRISMA guidelines. A computer-assisted literature search of PubMed and Ovid search engine was conducted. In order to increase the sensitivity of our search, we used free text words and MeSH terms with and without Boolean operators ("AND" and "OR"). Search terms keywords to identify different types of systemic and cutaneous lupus included "SLE", "systemic lupus erythematosus", "discoid lupus", "acute cutaneous lupus erythematosus", "subacute cutaneous lupus erythematosus", "discoid lupus" and "chronic cutaneous lupus erythematosus". We came across 85347 cases of different types of lupus when we used "OR" Boolean operator. We associated these 85347 studies with "Ustekinumab", "IL-12 inhibitor" and "IL-23 inhibitor" and the output was 12 studies. Out of these 12 only 5 studies fulfilled the inclusion criteria and were included in the study (Figure 2).

No language restrictions were enforced. The inclusion criteria for our systemic review were rather liberal. We included all the human studies that enrolled patients with different kinds of lupus treated with Ustekinumab. Other major inclusion criteria were minimum age of more than 18 years, SLE, CCLE and DLE patients who have received Ustekinumab. Exclusion criteria included review articles, basic science research, animal studies, irrelevant articles in which either the patient did not have any kind of lupus or in whom ustekinumab was not used for treatment. Following data were extracted and compared for all the studies: lupus type and subtype, age, gender, ethnicity, lupus clinical manifestations, ANA antibody levels, treatment duration and adverse reaction (Figure 3 and Table $1)$.

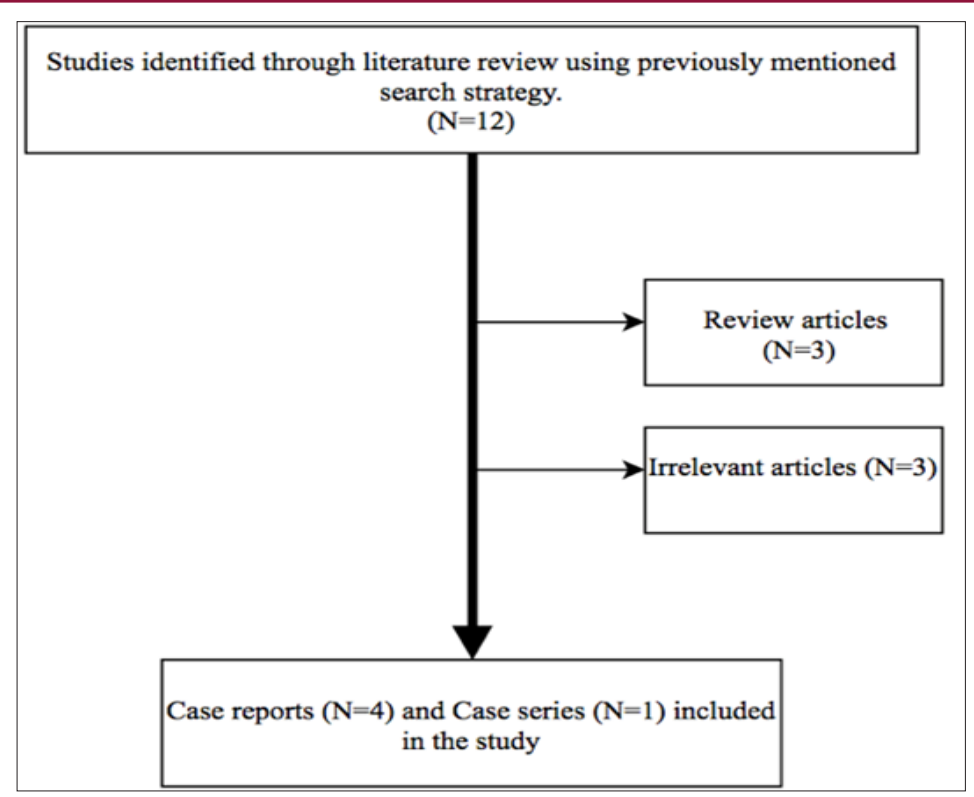

Figure 3: Search strategy for literature search.

\section{Discussion}

Psoriasis (Ps) is a relatively common disease affecting $1 \%$ to $3 \%$ of the US population, whereas SLE is significantly less common with reported prevalence rates in the United States ranging from 20 to 150 cases per 100,000 for SLE [11-12]. Cases of coexistent Ps and SLE are uncommon [13]. In one of the largest studies to date, it has been reported that cutaneous lupus manifestations (CLE) with or without SLE can occur in $0.69 \%$ of patients with Psoriasis and $1.1 \%$ of patients with CLE with or without SLE had concomitant psoriasis. Of these patients that had both, about 50\% had SLE of which $64 \%$ also had cutaneous lupus manifestations as part of SLE [14-15]. Three case reports of successful use of Ustekinumab in cutaneous lupus erythematosus without systemic involvement suggest that Th-17/ IL-23 may be involved in the pathogenesis of CLE $[9,10,16]$. Unlike our patient who had ongoing active SLE manifestations (thrombocytopenia), Chyuan et al reported a case of well controlled SLE and biopsy proven lupus nephritis, that developed resistant psoriasis, wherein Ustekinumab was successfully used and tolerated well, indicating its role in SLE [17].
In a recent retrospective chart review, of 96 patients with concomitant psoriasis and CLE with or without SLE (90\% of them had SLE), 5 patients were treated with Ustekinumab [15]. Four were maintained successfully on the drug, and one experienced loss of efficacy. Two of these patients had SLE with chronic plaque psoriasis, 2 had SLE, chronic plaque psoriasis and psoriatic arthritis, and 1 had discoid lupus erythematosus (DLE) with chronic plaque psoriasis. Improvement of all, cutaneous psoriasis, cutaneous SLE symptoms (malar and discoid rashes) lupus arthritis, oral ulceration, and hematologic abnormalities (thrombocytopenia or anemia) were reported among these patients. Although this study was not able to clearly discriminate between the improvements noted in the arthritis of psoriatic or lupus it showed that the patients on Ustekinumab had better outcomes. Although psoriasis and SLE have relatively different pathophysiologic mechanisms, they both share up regulation of the Th17 immune pathway with elevated levels of interleukin (IL)-17, IL-23, and IL-12 [18]. IL-17 \& IL-23 have been associated with the pathogenesis rheumatoid arthritis (RA), inflammatory bowel disease, ankylosing spondylitis and SLE 
[1-2]. Although not observed in all studies, serum IL- 17 levels have been found to be elevated in SLE compared to controls [19].

Correlations of serum IL-17 levels with SLE disease activity and anti-double stranded DNA (anti-dsDNA) antibody levels have previously been reported in some studies [5-6]. IL-12, elevated in SLE, stimulates the production of gamma interferon by TH-17 lymphocytes leading to glomerular nephritis and vasculitis. Serum p40 titers (IL-12 subunit) have been reported to be significantly higher in SLE patients compared to those of healthy subjects and patients with RA [20-21]. In SLE patients, serum p40 levels were positively correlated with the SLE disease activity index (SLEDAI) [21]. Ustekinumab binds and inhibits p40 subunit of interleukins
12 and 23, which are involved in the TH-17 signaling, this could be a potentially useful drug in the treatment of psoriatic arthritis with CLE or SLE. This case highlights the therapeutic challenges in managing a patient with concurrent psoriasis and SLE. Ustekinumab has been used frequently in treatment of psoriasis and psoriatic arthritis. However, its use is largely limited to a few case reports in patients with either CLE or in patients with psoriasis with coexistent CLE (Table 1). Herein, we report a patient with active psoriasis, active psoriatic arthritis and active SLE, who responded well to ustekinumab, there by suggesting the potential role of TH-17/ IL23 pathway as therapeutic target in treatment of cutaneous (non SLE) lupus and SLE. Further clinical studies are ongoing in order to investigate the role of Th-17 blocking agents in SLE.

Table 1: Overview of all the existing case reports of patients who have been treated with Ustekinumab for cutaneous lupus or SLE \& Psoriasis or cutaneous lupus or SLE alone.

\begin{tabular}{|c|c|c|c|c|c|c|c|c|}
\hline $\begin{array}{l}\text { Lupus } \\
\text { Type }\end{array}$ & Source & $\begin{array}{l}\text { Age/Gender/ } \\
\text { Ethnicity }\end{array}$ & Psoriasis & $\begin{array}{l}\text { Lupus } \\
\text { subtype }\end{array}$ & Antibody & $\begin{array}{c}\text { Other SLE } \\
\text { Manifestations }\end{array}$ & $\begin{array}{l}\text { Treatment } \\
\text { duration } \\
\text { (Months) }\end{array}$ & $\begin{array}{l}\text { Adverse } \\
\text { reaction }\end{array}$ \\
\hline \multirow[t]{4}{*}{ CCLE } & Varada et al [15] & $68 / \mathrm{F} / \mathrm{W}$ & PS/PsA & DLE & N/A & None & 36 & $\mathrm{~N} / \mathrm{A}$ \\
\hline & $\begin{array}{c}\text { Winchester at el } \\
{[16]}\end{array}$ & $41 / \mathrm{M}$ & Ps & DLE & ANA $(1: 1280)$ & None & $\mathrm{N} / \mathrm{A}$ & None \\
\hline & Dahl at el [10] & $79 / F$ & None & CCLE/DLE & None & None & 34 & None \\
\hline & De Souza at el [9] & $58 / \mathrm{F}$ & None & SCLE & ANA (1:640) & None & 12 & None \\
\hline \multirow[t]{6}{*}{ SLE } & Chyuan at el [17] & $21 / \mathrm{F}$ & Ps & SLE & $\begin{array}{c}\text { ANA Anti-Ds } \\
\text { DNA (171 IU/ } \\
\mathrm{ml})\end{array}$ & $\begin{array}{l}\text { Malar Rash, } \\
\text { Polyarthralgia } \\
\text { Ischemic stroke, } \\
\text { Lupus enteritis } \\
\text { Lupus nephritis }\end{array}$ & 10 & None \\
\hline & Varada et al [15] & 43/F/W & Ps/PsA & SLE & N/A & $\mathrm{N} / \mathrm{A}$ & 24 & $\mathrm{~N} / \mathrm{A}$ \\
\hline & Varada et al [15] & $38 / \mathrm{F} / \mathrm{B}$ & PS/PsA & SLE & N/A & N/A & 12 & Ps flare \\
\hline & Varada et al [15] & 25/F/B & PS/PsA & SLE & N/A & N/A & 1 & N/A \\
\hline & Varada et al [15] & $45 / \mathrm{F} / \mathrm{W}$ & Ps/PsA & SLE & N/A & N/A & 24 & Cellulitis \\
\hline & Our patient & $48 / \mathrm{M} / \mathrm{H}$ & Ps/PsA & SLE & $\begin{array}{c}\text { ANA Anti-Ds } \\
\text { DNA (48 IU/ } \\
\text { ml) }\end{array}$ & $\begin{array}{c}\text { Thrombocytopenia } \\
\text { AIHA }\end{array}$ & 36 & None \\
\hline
\end{tabular}

Abbreviations: M: male, F, Female, W: White, B: Black, H: Hispanic, N/A: Not available

\section{References}

1. Gaffen SL, Jain R, Garg AV, Cua DJ (2014) The IL-23-IL-17 immune axis: from mechanisms to therapeutic testing. Nat Rev Immunol 14(9): 585600 .

2. Sherlock JP, Taylor PC, Buckley CD (2015) The biology of IL-23 and IL17 and their therapeutic targeting in rheumatic diseases. Curr Opin Rheumatol 27(1): 71-75.

3. Chen DY, Chen YM, Wen MC, Hsieh TY, Hung WT, et al. (2012) The potential role of Th17 cells and Th17-related cytokines in the pathogenesis of lupus nephritis. Lupus 21(13): 1385-1396.

4. Wang Y, Ito S, Chino Y, Goto D, Matsumoto I, et al. (2010) Laser microdissection-based analysis of cytokine balance in the kidneys of patients with lupus nephritis. Clin Exp Immunol 159(1): 1-10.

5. Wong CK, Lit LC, Tam LS, Li EK, Wong PT, et al. (2008) Hyperproduction of IL-23 and IL-17 in patients with systemic lupus erythematosus: implications for Th17-mediated inflammation in auto-immunity. Clin Immunol 127(3): 385-393.

6. Vincent FB, Northcott M, Hoi A, Mackay F, Morand EF (2013) Clinical associations of serum interleukin-17 in systemic lupus erythematosus. Arthritis Res Ther 15(4): 97.
7. Wen Z, Xu L, Xu W, Xiong S (2014) Detection of dynamic frequencies of Th17 cells and their associations with clinical parameters in patients with systemic lupus erythematosus receiving standard therapy. Clin Rheumatol 33(10): 1451-1458.

8. (2012) Highlights of prescribing information: Stellara [Internet]: Food and Drug Administration.

9. De Souza A, Ali-Shaw T, Strober BE, Franks AG (2011) Successful treatment of subacute lupus erythematosus with ustekinumab. Arch Dermatol 147(8): 896-898.

10. Dahl C, Johansen C, Kragballe K, Olesen AB (2013) Ustekinumab in the treatment of refractory chronic cutaneous lupus erythematosus: a case report. Acta Derm Venereol 93(3): 368-369.

11. Kurd SK, Gelfand JM (2009) The prevalence of previously diagnosed and undiagnosed psoriasis in US adults: results from NHANES 2003-2004. J Am Acad Dermatol 60(2): 218-224.

12. Pons-Estel GJ, Alarcón GS, Scofield L, Reinlib L, Cooper GS (2010) Understanding the epidemiology and progression of systemic lupus erythematosus. Semin Arthritis Rheum 39(4): 257-268.

13. Millns JL, Muller SA (1980) The coexistence of psoriasis and lupus erythematosus. An analysis of 27 cases. Arch Dermatol 116(6): 658-663. 
14. Zalla MJ, Muller SA (1996) The coexistence of psoriasis with lupus erythematosus and other photosensitive disorders. Acta Derm Venereol Suppl (Stockh) 195: 1-15.

15. Varada S, Gottlieb AB, Merola JF, Saraiya AR, Tintle SJ (2015) Treatment of coexistent psoriasis and lupus erythematosus. J Am Acad Dermatol 72(2): 253-260.

16. Winchester D, Duffin KC, Hansen C (2012) Response to ustekinumab in a patient with both severe psoriasis and hypertrophic cutaneous lupus. Lupus 21(9): 1007-1010.

17. Chyuan IT, Tsai TH, Chang TH, Wu CS (2014) Ustekinumab treatment in a patient with psoriasis and systemic lupus erythematosus. Lupus 24(6) 650-651.
18. Du J, Li Z, Shi J, Bi L (2014) Associations between serum interleukin-23 levels and clinical characteristics in patients with systemic lupus erythematosus. J Int Med Res 42(5): 1123-1130.

19. Rana A, Minz RW, Aggarwal R, Anand S, Pasricha N, et al. (2012) Gene expression of cytokines (TNF- $\alpha$, IFN- $\gamma$ ), serum profiles of IL-17 and IL-23 in pediatric systemic lupus erythematosus. Lupus 21(10): 11051112.

20. Koenig KF, Groeschl I, Pesickova SS, Tesar V, Eisenberger U, et al. (2012) Serum cytokine profile in patients with active lupus nephritis. Cytokine 60(2): 410-416.

21. Lauwerys BR, Van Snick J, Houssiau FA (2002) Serum IL-12 in systemic lupus erythematosus: absence of p70 heterodimers but presence of p 40 monomers correlating with disease activity. Lupus 11(6): 384-387.



